\title{
Cellular innate and adaptive immunity are affected by chronic exercise: implication of the cannabinergic system
}

3 Salvador Valencia-Sánchez ${ }^{1}$, Karen Nava-Castro ${ }^{2 \uparrow, ~ M . ~ I s a b e l ~ P a l a c i o s-A r r e o l a ~}{ }^{2 \uparrow}$, Oscar Prospéro-

4 García $^{3 \&}$, Jorge Morales-Montor*4, and René Drucker-Colín ${ }^{1 \& \dagger}$

$5 \quad{ }^{1}$ Instituto de Fisiología Celular, Departamento de Neuropatología Molecular, Universidad Nacional

6 Autónoma de México, Circuito Exterior s/n, Ciudad Universitaria, Ciudad de México, México

$7 \quad{ }^{2}$ Genotoxicología y Mutagénesis Ambientales, Ciencias Ambientales, Centro de Ciencias de la

8 Atmósfera, Ciudad de México, México.

9 32Laboratorio de Cannabinoides, Departamento de Fisiología, Facultad De Medicina, Universidad

10 Nacional Autónoma de México, Circuito Exterior s/n, Ciudad Universitaria, Ciudad de México,

11 México

$12{ }^{4}$ Departamento de Inmunología, Instituto de Investigaciones Biomédicas, Universidad Nacional

13 Autónoma de México, AP 70228, 04510 Ciudad de México, México.

15 Corresponding author

16 * Jorge Morales-Montor \& René Drucker-Colín

17 E-mail: jmontor66@biomedicas.unam.mx,

19 These authors contributed equally to this work.

20 \&These authors also contributed equally to this work. 


\section{Abstract}

22 The impact of performing exercise on the immune system presents contrasting effects on health when

23 performed at different intensities. In addition, the consequences of performing chronic exercise have

24 not been sufficiently studied in contrast to the effects of acute bouts of exercise. Our findings shed

25 light on the effects that chronic exercise elicits on several immune cell subpopulations, from the

26 innate to the adaptive immunity. For this study male Wistar rats performed treadmill running 5 times

27 a week for a period of 10 weeks, speed and duration in each exercise bout was gradually increased

28 until reaching 40 minutes at $15 \mathrm{~m} / \mathrm{min}$. Our results showed a significant decrease in lymphocyte

29 subpopulations (CD4+, $\mathrm{T} \gamma \delta$, and $\mathrm{CD} 45 \mathrm{RA}+$ cells) and also indicate an alteration in the cannabinoid

30 receptors expression in some of these cells subsets. Although functional assays did not reveal any

31 variation in total immunoglobulin production or NK cells cytotoxic activity, proliferative capability

32 of total splenocytes increased in trained rats. Our results further support the notion that exercise

33 affects the immunological system and extends the description of underlying mechanisms mediating

34 such effects. Altogether, our results contribute to the understanding of the benefits of exercise on the

35 practitioner's general health.

\section{Introduction}

38 The beneficial impact of exercise on the practitioner's general health is a well-known fact [1-3].

39 Studies suggesting a connection between physical activity and the improvement of health have

40 generated information that ranges from describing adipose tissue loss to changes in genetic

41 expression [4,5]. It is well known that the immune system (IS) is critical to maintain health, and

42 whether it is enhanced or hindered when performing exercise is still controversial. Its proper 
43 composition and its correct function will allow it to actively overcome challenges that otherwise

44 would compromise the organism's health such as infections, autoimmune diseases, cancer, etc.

46 Data regarding the changes induced by exercise in cell subpopulations of the IS and their function

47 seems to be controversial [6-8]. This may be partly explained by the use of different exercise

48 paradigms. Along with this idea, many studies document the immediate changes on the IS induced by

49 a single bout of exercise, as opposed to the effects of its chronic performance, which suggest

50 different outcomes and in some cases opposite effects over the IS [8-10]. Less attention has been

51 paid to such studies and to the long-term alterations that it may produce on the IS. For instance,

52 macrophages extracted from mice trained for 12 weeks exhibited increased phagocytic activity,

53 superoxide anion production and glucose consumption when compared to macrophages obtained

54 from sedentary mice [8]. Consistently other studies have shown that chronic exercise alters the

55 function of $\mathrm{T}$ cells, affecting their production of pro- and anti-inflammatory cytokines, including the

56 up-regulation of IL-2, an important cytokine related to proliferation and activation [10]. Likewise,

57 trained rats presented increased glucose consumption, IL-2 production and IL-2R expression by their

58 lymphocytic subpopulations. Furthermore, those changes obtained in trained animals seem to last

59 days after the last exercise bout [10].

61 Many molecular pathways that are affected by exercise possess an immunoregulatory potential,

62 ranging from variations in the energy substrates $[11,12]$ to the activation of signaling pathways with

63 direct immune-regulatory relevance, such as: the release of IL 6 by skeletal muscle [4,9], release of

64 stress hormones, catecholamines $[4,13]$ and neurotransmitters by the sympathetic and para-

65 sympathetic system, among others. Hence, in order to contribute to the further understanding of these 
66 effects we decided to evaluate the cannabinergic system (CBS). Regarding this system, some studies

67 have reported a subtle increase of anandamide, a widely studied molecule that acts as a CB1 and CB2

68 receptor agonist, after short bouts of aerobic exercise. Such increase was sustained up to several

69 minutes after the conclusion of the physycal activity [14-16]. Furthermore, both receptors are widely

70 distributed in the immune cells and IS structures [17-19] and when activated, together or

71 independently, produce changes in the function of several immune cells, suggesting that their

72 activation is able to modulate the IS function. These modulatory actions have been explored in vitro

73 [20,21] and in vivo [19]. Likewise, the expression of cannabinergic receptors (CBR) on the surface of

74 immune cells, varies according to their activation and inflammatory status. Given that new data

75 suggests its relevance as an immune-modulatory system, the expression of these receptors provides

76 us with interesting and relevant information about the IS status.

77 Altogether, our study is focused on exploring the long-term changes that chronic exercise (CE)

78 produces in the proportion of splenocytes from the adaptive and innate immunity, assessing the

79 effects of $\mathrm{CE}$ on the function of splenocytes (by performing proliferation tests and cytotoxicity test

80 with total splenocytes in vitro), and finally on determining if the expression of CBR in immune

81 cells is affected by chronic exercise. Our findings suggest a wide variety of effects induced by CE on

82 several immune cell subpopulations, from the innate and adaptive immunity. The latter being the

83 most affected by CE, with significant decreases in some lymphocyte subpopulations (CD4+ and

84 CD45 RA+ cells). Our results also suggest an alteration in the expression of CBR as a consequence

85 of training in natural killer cells (NK), T helpers, T $\gamma \delta$ lymphocytes and B lymphocytes. Surprisingly,

86 functional assays did not reflect any impairment in total immunoglobulin production or cytotoxic

87 activity from splenocytes derived from trained rats, which is contrasting with the decrease in

88 lymphocytic subpopulations. Furthermore, results from the proliferation assay showed a significant 
89 enhancement in the proliferative capabilities of splenocytes from trained rats, opening a new

90 perspective on the boosted efficiency in immune cell subpopulations promoted by CE.

91

\section{Materials and Methods}

\section{Ethic statement}

94 Animal care and experimental practices were conducted at the Animal Facilities of the Instituto de

95 Fisiología Celular (IFC), Universidad Nacional Autónoma de México (UNAM). All procedures in

96 the experimental animals were approved by the Institutional Care and Animal Use Committee

97 (CICUAL), adhering to Mexican regulation (NOM-062-ZOO-1999), in accordance with the

98 recommendations from the National Institute of Health $(\mathrm{NIH})$ of the United States of America (Guide

99 for the Care and Use of Laboratory Animals). Euthanasia of experimental animals was performed in

100 a humanitarian way.

\section{Animals}

103 For this study, male Wistar rats ranging between 250 to $300 \mathrm{~g}$ were used, proceeding from our own

104 breeding at the animal facilities of IFC, UNAM. The animals were housed at IFC with controlled

105 temperature $\left(22^{\circ} \mathrm{C}\right)$ and 12 -hr light-dark cycles, with water and Purina LabDiet 5015 chow ad libitum

106 (Purina, St. Louis MO). Rats were sacrificed by cervical dislocation after pentobarbital sodium

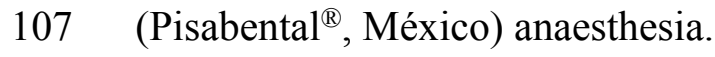




\section{Exercise Protocol}

110 Animals were set in one of three experimental groups: Exercised (EXE), Treadmill control (TC) and

111 sedentary group (SED). Animals in the exercised group performed treadmill running 5 times a week

112 for a period of 10 weeks, for which a previous habituation of one week was completed. During the

113 habituation week, animals were placed inside the treadmill and then it was turned on at minimum

114 capacity $(4 \mathrm{~m} / \mathrm{min})$ for 5 minutes per day. Once the habituation period was completed, animals started

115 training. On the first day of training, rats ran at $7.5 \mathrm{~m} / \mathrm{min}$ for 10 minutes, then speed and duration of

116 exercise was escalated gradually each consecutive day, in order to achieve a daily exercise bout of 40

117 minutes at $15 \mathrm{~m} / \mathrm{min}$ by the fourth week. Remaining weeks of training were kept constant in speed

118 and duration until the sacrifice of the animals.

119 Animals sited in the TC group were placed inside the treadmill at minimum capacity $(4 \mathrm{~m} / \mathrm{min})$ for 10

120 minutes, 5 times per week, for the same period of time than the exercised group (10 weeks). While

121 being inside the treadmill, animals from TC group were exposed to the same context than animals

122 from the EXE group without being exercised, reflecting any effect in the results prompted by

123 sources other than exercise itself. Animals conforming the SED group were kept alive in standard

124 conditions for the same amount of time than the other two groups.

125 At the end of the training period animals from every group were allowed to rest for two days in order

126 to eliminate any possible effect of acute exercising. Afterwards, animals were sacrificed and samples

127 taken.

\section{Flow Cytometry}

129 Spleens were manually disaggregated using a 50 $\mu$ m nylon mesh, and cells resuspended in PBS.

130 Erythrocytes in the solution were lysed using ACK buffer $\left(150 \mathrm{mM} \mathrm{NH}_{4} \mathrm{Cl}, 10 \mathrm{mM} \mathrm{KHCO}, 0.1 \mathrm{mM}\right.$ 
$131 \mathrm{Na}_{2}$ EDTA, $\mathrm{pH}$ 7.3) for 10 minutes and washed three times with PBS, then cells were resuspended in

132 FACS buffer (PBS, FBS, $\left.0.02 \% \mathrm{NaN}_{3}\right)$.

133 Approximately $1 \times 10^{6}$ cells were incubated with the following antibodies in order to characterize

134 spleen immune subpopulations: Alexa Fluor ${ }^{\circledR}$ 488-conjugated- anti rat CD3 (IF4, Biolegend), PE

135 Cy5-conjugated- anti rat CD4 (biolegend), PE-conjugated anti rat CD8a (Biolegend), PE-conjugated

136 -anti rat CD45RA (Biolegend), Alexa Fluor ${ }^{\circledR}$ 647-conjugated- anti rat CD161 (biolegend), biotin-

137 conjugated- anti rat CD11b (OX-42, Bioloegend), PE-conjugated anti rat TCR (V65, Biolengend).

138 For the staining of cannabinoid receptors, the polyclonal primary antibodies used were: rabbit anti

139 Cannabinoid receptor I $\left(\right.$ abcam $\left.^{\circledR}\right)$ and rabbit anti Cannabinoid receptor II $\left(\right.$ abcam $\left.^{\circledR}\right)$, followed by the

140 Secondary antibodies: AlexaFluor ${ }^{\circledR}$ 488- conjugated goat anti rabbit IgG (ThermoFisher Scientific)

141 and DyLight ${ }^{\circledR}$ 649- conjugated- anti rabbit IgG (Vector laboratories).

142 In order to assess proliferation, a Cell Trace ${ }^{\mathrm{TM}}$ CFSE cell proliferation kit was used (Invitrogen ${ }^{\mathrm{TM}}$ ).

143 Propidium iodide was used to determine viability of cells during cytotoxic assays. Attune Cytometer

144 (life technologies) was used to obtain data which was further analyzed with FlowJo software

145 (Treestar Inc.).

\section{Proliferation assays}

148 Total splenocytes were obtained as previously described and quantified on a Neubauer chamber.

149 Subsequently, splenocytes were marked with CFSE cell tracer, , which was used according to the

150 manufacturer's protocol. Finally, cells were resuspended in RPMI-1640 medium (ATCC ${ }^{\circledR} 30$

$1512001^{\mathrm{TM}}$ ). Splenocytes were cultivated in RPMI-1640 medium plus Ionomicyn (SIGMA-ALDRICH ${ }^{\circledR}$ 
152 10634 ${ }^{\mathrm{TM}}$ ) and PMA (SIGMA-ALDRICH ${ }^{\circledR}$ P8139-1MG ${ }^{\mathrm{TM}}$ ) at concentrations100 $\mathrm{nm}$ and and 25ng/ml

153 for 72 hours. Proliferation was assessed using an Attune cytometer (Life Technologies) with blue and

154 red lasers, obtained data was further analyzed with FlowJo software (Treestar Inc.).

\section{Cell Culture}

157 Yac 1 cell line (ATCC ${ }^{\circledR}$ - TIB 160 ${ }^{\mathrm{TM}}$ ) was cultivated in RPMI 1640 medium (ATCC ${ }^{\circledR}$ 30-2001 ${ }^{\mathrm{TM}}$ )

158 supplemented with 10\% Fetal bovine serum (FBS, ATCC® 30-2020 ${ }^{\mathrm{TM}}$ ) and kept with air, 95\%;

159 carbon dioxide $\left(\mathrm{CO}^{2}\right), 5 \%$ at $37^{\circ} \mathrm{C}$. Yac 1 cells were expanded for a week then counted on a

160 Neubauer chamber and finally stained with CFSE kit for further use in cytotoxic assays.

\section{In vitro cytotoxic assay}

163 Yac 1 cells were stained with cell trace ${ }^{\mathrm{TM}}$ CFSE kit according to the manufacturer's protocol and

164 used as target cells for the assay. A single cell splenocyte suspension was obtained from rats in the

165 different experimental conditions as previously described, they were counted and used as the effector

166 cells in the assay. Finally both, effector and target cells were co-cultivated in RPMI medium

167 supplemented with 10\% FBS for 4 hours, into 96 round well plates. Thereafter co-cultures were

168 removed from the incubator and stained with Propidium Iodide and washed with Facs buffer.

169 Acquisition was performed in an Attune Cytometer (life technologies) and data further analyzed

170 with FlowJo software (Treestar Inc.). 


\section{Corticosterone and IgG levels assesssment}

173 Animals were anesthetized and sacrificed one day after concluding their experimental condition, at

174 the same time that they had been set for exercising (14:00-16:00 hr.). Cardiac puncture was

175 performed in order to extract blood, which was immediately centrifuged at 4000rpm to collect the

176 blood serum. Subsequently, blood serum was divided into two aliquots and stored at $-70^{\circ} \mathrm{C}$ for later

177 use. Corticosterone levels were assessed with a Corticosterone ELISA kit (Abcam ${ }^{\circledR}$ ab-108821) and

178 procedures underwent according to manufacturer's protocol. Assessment of the levels of IgG in

179 serum were carried out on a 96 flat bottom well plate. The plate was previously sensitized with a

180 dilution of blood serum (1:1000), washed and blocked with a 1\% albumin solution. Subsequently, ,

181 the plate was incubated for 2 hours antibodies $\alpha$ IgG rat HRP were incubated, and once incubation

182 finished, several washes were performed, chromogen was added to the wells, the reaction was

183 stopped and the reading of the plate was carried out on a Stat Fax 4200 microplate reader (Awarness

184 Technology).

\section{Statistical analysis}

187 For data regarding the changes of every cell subpopulation, a one-way ANOVA $(\alpha=0.05)$ was performed followed by a Tukey post-hoc test. Differences were considered significant when $\mathrm{p}<0.05$,

189 with the actual $\mathrm{p}$ value and $\mathrm{n}$ being stated in each figure legend. Before the selection of the ANOVA

190 test the normal distribution of the data was assessed via Shapiro-Wilk test. A similar process was

191 carried out for the statistical analysis of the data regarding the proliferation and cytotoxicity tests, as

192 well as for the data concerning the levels of corticosterone and IgG in serum. For the assessment of

193 the expression of CBR, a two-way ANOVA $(\alpha=0.05)$ was performed, because of the consideration 
194 of two independent variables (group and CBR), followed by a Bonferroni post-hoc test with the same

195 significant difference criterion. Data from all the experiments were charted as mean \pm standard error, 196 and analysed with Prism 5 software for Mac (GraphPad Software Inc.)

\section{Chronic-moderate exercise alters the composition of splenocyte}

\section{0 subpopulations}

201 Distribution of immune cells is a parameter that provides information about deficiencies or

202 alterations from the IS, therefore we decided to evaluate several cell subpopulations from the innate

203 and adaptive immune system in the spleen of rats that underwent different experimental conditions

204 (supplementary Fig 1). The immune cell subpopulations from the innate immune response that were

205 analyzed corresponded to: NK cells (CD161+) and macrophages (CD11b+) for which flow cytometry

206 analysis did not reflect a significant difference among groups (Fig 1). Analyzed cells from the

207 adaptive immune response were: total T lymphocytes (CD3+), T helper lymphocytes (CD4+),

208 cytotoxic T lymphocytes, $\mathrm{T} \gamma \delta$ lymphocytes $(\mathrm{T} \gamma \delta+)$ and B lymphocytes (CD45 RA+). For those cells

209 studied, flow cytometry analysis reflected a decrease in the proportion of $\mathrm{T}$ helper lymphocytes and

210 in B lymphocytes from the EXE group when compared to both control groups, SED and TC (Figs 2

211 and 3), therefore considering such changes an effect of CE. On the other hand $\mathrm{T} \gamma \delta$ lymphocytes

212 showed an increase in the EXE group when compared to SED and TC groups (Fig 2), once more

213 reflecting a change attributable to training. On the other hand, T cytotoxic cells decreased in TC and

214 EXE groups in contrast to SED control group, reflecting an effect non attributable to exercise, but to 
215 the exposure to the treadmill (Fig 2). Total T lymphocytes (CD3+), did not show changes among the

216 experimental groups (Fig 2).

response.

(A) Representative dot plot of the cytometric analysis of the subpopulation percentages. (B)

Determination of splenocyte subpopulations from the innate immune response one day after being exposed to each condition in the different groups: SED, TC and EXE; data from 4 independent experiments are expressed as mean $\pm \mathrm{SE}$. No subpopulation showed statistically significant changes: Natural killer cells (ANOVA, $\mathrm{p}=0.0683, \mathrm{n}=10$ ), and Macrophages (ANOVA, $\mathrm{p}=0.0273, \mathrm{n}=10$ ).

231 that showed statistically significant changes were: $T$ helper lymphocytes (ANOVA, $\mathrm{p}=0.0008, \mathrm{n}=5$ )

232 and $\mathrm{T} \gamma \delta$ lymphocytes (ANOVA, $\mathrm{p}=0.0002, \mathrm{n}=10$ ). T lymphocytes (ANOVA, $\mathrm{p}=0.3739, \mathrm{n}=12$ ),

233 cytotoxic T lymphocytes (ANOVA, $\mathrm{p}=0.0016, \mathrm{n}=12$ ). * Means statistically different from the two 234 other groups, $\mathrm{P}<0.05$. 
236 (A) Representative dot plot of the cytometric analysis of the subpopulation percentages. (B)

237 Determination of B-lymphocytes of spleen of rats in the different groups: SED, TC and EXE; data

238 from 4 independent experiments are expressed as mean \pm SE. B lymphocytes showed a statistically

239 significant change (ANOVA, $\mathrm{p}=0.3601, \mathrm{n}=10$ ). $*$ Means statistically different from the two other

240 groups, $\mathrm{P}<0.05$.

243 Modulation of CBR Expression in splenocytes after chronic exercise

244 CBR are widely distributed among immune cell subpopulations and structures from the IS. The expression of CBR on immune cells has been demonstrated to vary depending on activation or

246 inflammatory profile, among other parameters. Thus, we decided to evaluate if CE would promote 247 changes in the expression of CBR on splenocytes.

248 From the innate immune system, NK cells (CD161+) and macrophages were analyzed. NK cells from

249 EXE and TC groups showed a decrease in CB2 expression, compared to SED, while no change was

250 observed in $\mathrm{CB} 1$ expression. Macrophages $(\mathrm{CD} 11 \mathrm{~b}+)$ did not present changes in the expression of

251 any CBR among groups (Fig 4). From the adaptive immune response, T helper lymphocytes (CD4+

252 cells) presented an increase in the expression of CB1 in animals from EXE group when compared to

253 both control groups SED and TC, while no statistically significant difference was observed regarding

$254 \mathrm{CB} 2$ expression. A similar phenomenon was observed in $\mathrm{T} \gamma \delta$ subpopulation from EXE animals,

255 which showed a higher expression of CB1 when compared to SED and TC groups (Fig 5), with no

256 change in the expression of CB2. The expression of CBR did not vary in the subpopulations of T 
257 lymphocytes (CD3+) and cytotoxic T lymphocytes when experimental groups were compared (Fig

$2585)$.

Fig 4. Expression of CBR in splenocyte populations related to the innate immune response.

260 Analysis of the expression of CB1 and CB2 CBR in splenocyte populations from the innate immune

261 response (NK's and macrophages) among experimental groups: SED (shaded bar), TC (white bar)

262 and EXE (Solid bar); data from 4 independent experiments are expressed as mean \pm SE. Lines

263 connecting bars represent comparison among groups, ${ }^{*} \mathrm{p}<0.05$. Two way ANOVA and Bonferroni

264 post-test, $\mathrm{n}=9$.

Fig 5. Expression of CBR in splenocyte populations related to the adaptive immune response.

Analysis of the expression of $\mathrm{CB} 1$ and $\mathrm{CB} 2 \mathrm{CBR}$ in splenocyte populations from the adaptive

immune response ( $\mathrm{T}$ lymphocytes; $\mathrm{n}=10$, T helper lymphocytes; $\mathrm{n}=8$, cytotoxic $\mathrm{T}$ Lymphocytes;

$\mathrm{n}=10$ and $\mathrm{T} \gamma \delta \mathrm{n}=8$ ) among experimental groups: SED (shaded bar), TC ( white bar) and EXE (Solid

bar); data from 4 independent experiments are expressed as mean $\pm \mathrm{SE}$. Lines connecting bars

270

represent comparison among groups, ${ }^{*} \mathrm{p}<0.05$. Two way ANOVA and Bonferroni post-test.

On the other hand, B lymphocytes from the spleen of EXE animals showed a significant increase in CB2 expression, compared to those from SED and TC groups, whilst no change was reflected

274 between groups in the expression of CB1 (Fig 6).

275 Fig 6. Expression of CBR in B lymphocytes from spleen.

276 Analysis of the expression of CB1 and CB2 CBR in B lymphocytes from spleen among experimental

277 groups: SED (shaded bar), TC (white bar) and EXE (Solid bar); data from 4 independent experiments 
are expressed as mean \pm SE. Lines connecting bars represent comparison among groups, ${ }^{*} \mathrm{p}<0.05$.

279 Two way ANOVA and Bonferroni post-test, $\mathrm{n}=10$.

\section{Immunoglobulin $G$ levels are not altered by chronic exercising}

282 Immunoglobulin $\mathrm{G}(\mathrm{IgG})$ is the most abundant type of immunoglobulins and a reliable parameter to assess the function of plasmatic cells. A change in the amount of IgG could represent an ongoing infectious process or an alteration on the normal function of plasmatic cells when observed in intact animals. We decided to assess if the total production of IgG would vary among our experimental groups. To do so, we performed a direct semi-quantitative ELISA. When data was analyzed experimental groups: SED (optic density 2.731),TC and EXE (OD 2.973 and 2.871 respectively, supplementary Fig 2).

\section{Chronic exercise enhances proliferative capacity but not cytotoxic}

\section{activity of total splenocytes}

294 Proliferative capacity and cytotoxic activity have been tested before in order to assess the degree of 
total splenocytes from EXE group showed a higher proportion of dividing cells when compared to

300 SED and TC groups (Fig 7C). Furthermore, we decided to test the proliferative capacity of the

301 immune cell subpopulations and we found that B lymphocytes and NK cells from the EXE group

302 exhibited a higher proportion of dividing cells than both SED and TC groups (Figure 7D).

303 Fig 7. Effect of chronic exercise on splenocyte proliferation.

304 (A) Representative histograms of cytometric analysis of dividing cells. Two parameters were

305 considered to assess the proliferative capacity of splenocytes: (B) the proliferation index, which did

306 not change due to chronic exercise and (C) the proportion of dividing cells, where we observed an

307 increase in the exercised group when compared to both control groups (ANOVA, $\mathrm{p}=0.0092, \mathrm{n}=5$ ).

308 (D) Analysis of proliferative capacity of specific splenocyte subpopulations: T helper lymphocytes

309 (ANOVA, $\mathrm{p}=0.4543, \mathrm{n}=5$ ) and cytotoxic $\mathrm{T}$ lymphocytes (ANOVA, $\mathrm{p}=0.5248, \mathrm{n}=6$ ) did not show

310 any change between groups; $B$ lymphocytes (ANOVA, $\mathrm{p}=0.0006, \mathrm{n}=6)$ and Natural killers (ANOVA,

$311 \mathrm{p}=0.0191, \mathrm{n}=6)$ from EXE did show an in increase in the proportion of dividing cells when compared

312 to both control groups. In graphic bars SED is represented by shaded bar, TC by white bar and EXE

313 by the solid bar.

315 In order to measure the cytotoxic capacity of splenocytes from the different groups, we performed a

316 cytotoxicity test utilizing Yac1 cells as target cells and cultivating them with total splenocytes from

317 animals of the different groups. Yac1 cells were previously marked with CFSE, and at the end of the

318 test dead cells were dyed with propidium iodide, so double positive cells represented the target cells

319 killed. The assay was carried out at three different effector/target ratios: 10:1, 50:1 and 100:1 and

320 none showed any significant difference among treatments after the statistical analysis (Fig 8). 
321 Fig 8. Cytotoxic activity of total splenocytes in vitro.

322 (A) Representative dot plot of cytometric analysis of killed target cells percentage. (B) Cytotoxic

323 activity of total splenocytes was assessed in vitro at three different ratios (effector cells: target cells,

324 10:1, 50:1 and 100:1); data from 2 independent experiments are expressed as mean \pm SE, with an $n=6$

325 for each condition. No differences were found among the groups at any of the different ratios. In bar

326 graphics SED is represented by the shaded bar, TC by the white bar and EXE by the solid bar.

\section{Corticosterone level is not altered by chronic exercise}

330 Corticosterone level is considered a reliable stress marker in animals. In order to further comprehend

331 the data obtained, we decided to analyze if corticosterone levels of blood serum differ among groups,

332 reflecting a possible long-term effect of stress in exercised animals. When data was statistically

333 analyzed (ANOVA, $n=6, p=0.0473$, Tukey’s) results show a significant difference among the

334 experimental groups. Nonetheless, The post hoc analysis did not show any significant interaction

335 among the experimental groups. Means of the groups: TC and EXE (102.6 and 96.5 ng/ml

336 respectively) were notoriously higher than that of the SED group (46.8 ng/ml, supplementary Fig 3). 
339 Although widely studied, the consequences of physical activity over the IS remain as a promising

340 field not only to expand our comprehension on basic physiology, but also on physiopathology and the

341 different processes that take part on the orchestration of the immune response. At first instance, our

342 investigation tries to emulate in rats a popular paradigm of exercising in modern times across the

343 global population, which involves the chronic performance of medium intensity resistance exercise.

344 Such particularities in our model have led us to asses its raw impact over the IS composition and its

345 function. Notwithstanding, the vast majority of studies have focused in the effects of short bouts of

346 exercise over the immediate changes in composition and function of the IS [1,8,22,23], and

347 neglecting those focused on the effect of CE and its long lasting effects over the IS [9,24]. In this

348 study we observed that components from the innate immune response were not affected by chronic-

349 moderate exercise, while elements from the adaptive immune response did change in those animals

350 that underwent physical training. T helper lymphocytes and B lymphocytes were decreased in trained

351 animals, contrary to what would be expected according to the popular statement of moderate exercise

352 enhancing a pro-inflammatory state $[25,26]$. On the other hand, $T \gamma \delta$ lymphocytes increased in

353 animals from the EXE group, augmentation that could reflect an increased surveillance and

354 protection of the upper respiratory tracts (URT) and mucosa tissue, idea that would be in accordance

355 with the strengthened resistance against URT infections due to moderate exercising and opposite to

356 the well documented effect of higher susceptibility to these infections in high performance athletes

$357[23,27-31]$. Nonetheless, the reduction of T helper and B lymphocyte populations was unexpected,

358 since these cells play a major role at recognizing antigens and therefore at orchestrating immune

359 responses against new and already known threats and once activated they can polarize towards a pro-

360 inflammatory or anti inflammatory state which modulates the activity of several other components

361 from the innate and adaptive immune response. Consequently, the decrease of T helper lymphocytes

362 as well as that of B lymphocytes made us wonder if it could be translated into a deficient immune

363 response of animals of the EXE group. 
364 Once we determined that our exercising paradigm did affect the composition of immune cells

365 subpopulations, our next goal became to assess if those changes would be translated into functional

366 alterations. To accomplish our purpose, we determined total IgG in serum among experimental

367 groups and we also performed proliferation and cytotoxicity test in vitro. On the proliferation test,

368 two parameters were evaluated: the proliferation index and the percentage of dividing cells. The first

369 represents the mean of divisions that dividing cells underwent during the assay, which showed no

370 difference among groups. In turn, the percentage of dividing cells among groups during the

371 experiment showed statistical differences, being higher in splenocytes from the EXE group.

372 Altogether, this data suggests that cells from EXE group are not more efficient at dividing once they

373 have been activated but that more cells in proportion from EXE group are prone to proliferate once

374 they have been exposed to PMA and ionomycine. These results made us wonder if this trend would

375 be persistent in basal conditions, so we compared the percentage of proliferation in splenocytes

376 among the experimental groups without activation by PMA and ionomycine and the statistical

377 analysis showed no difference among them, reflecting a response produced by activation and not an

378 anomaly that could reflect an inflammatory state that in turn could favor an autoimmune response.

379 Thereafter, we evaluated the percentage of dividing cells from specific subpopulations, $\mathrm{T}$ helper

380 cells, cytotoxic T cells, B lymphocytes and NK cells, showing an increase in the percentage of

381 dividing cells in the last two subpopulations by effect of exercise. On the other hand, statistical

382 analysis from the cytotoxicity test did not show variation among the experimental groups in any of

383 the target/effector cell ratios tested, so data provided from this experiment does not suggest a higher

384 cytotoxic activity from NK cells as a consequence from exercise. Nonetheless, exercise enhances the

385 amount of NK cells that proliferate, which may indicate a higher immune-surveillance against

386 transformed and virally infected cells in chronically exercised subjects. 
387 Even though we found a decrease in major lymphocyte subpopulations (CD4+ and CD45 RA+ cells),

388 which has been reported before for other immune cells in long term exercised individuals [26,32-34],

389 we also determined that a bigger proportion of splenocytes is prone to activate when stimulated, plus

390 other functions of splenocytes from the EXE animals were not impaired. Given the fact that long-

391 term exercised subjects do not report any kind of immune suppression, we have come to hypothesize

392 that the decrease in the composition of some cell subpopulations may represent a more efficient IS,

393 which requires less elements but presents a stronger reaction when needed. This idea is also

394 supported by the results shown by the ELISA test, which showed that levels of IgG do not change

395 among the groups, even though the subpopulation of B lymphocytes is decreased in EXE animals.

396 Finally, recent works have denominated the endo-cannabinoid system (ECS) as an

397 immunomodulatory system; inhibiting the function of highly reactive and pro-inflammatory cells

$398[21,35,36]$. The ECS exerts its functions through the activation of its receptors, which expression

399 vary greatly depending on cell subpopulation, activation or inflammatory status [20,35,37,38], being

400 increased in the surface of more reactive cells. In this work we demonstrated that CE promotes

401 changes in the expression of CBR's in splenocytes, findings that concur with previous works

402 showing that during moderate exercise bouts there is an increase of circulating ECS agonists [14,15].

403 We must emphasize that changes in CBR's expression remained after one skipped day of training,

404 which suggests that subjects exercising on a daily basis or in intervals of every two days might be

405 maintaining this alterations. Our methodology allowed us to determine differences in the expression

406 of CBR among cell subpopulations even in both control groups. These differences might be

407 explained by the intrinsic variability among cell subpopulations. We also assessed an increased

408 expression of CB1 receptor in the subpopulations of T helper and $\mathrm{T} \gamma \delta$ lymphocytes and an increase in

409 the expression of CB2 receptor in B lymphocytes of exercised animals. Therefore enhanced

410 expression of $\mathrm{CB} 1$ in $\mathrm{T}$ helper, $\mathrm{T} \gamma \delta$ and $\mathrm{CB} 2$ in $\mathrm{B}$ lymphocytes could represent a mechanism to 
411 diminish the activity of these highly metabolic and inflammatory cells from EXE animals, as

412 reflected by the proliferation tests. Some of these data differ from anterior reports concerning

413 expression of CBR, nonetheless most of those reports used different techniques and did not measure

414 the protein conforming CBR's $[17,39,40]$.

415 We would like to address those changes presented on this work that can not be attributable to CE,

416 like the composition of cytotoxic T cells for which TC and EXE groups differ when compared

417 against SED group, reflecting an effect relying probably on the stress produced by the placement of

418 the animals inside the treadmill, being that, the one thing that those groups had in common. The same

419 explanation seems plausible for the expression of CBR in NK and cytotoxic T cells. Nevertheless

420 analysis of corticosterone did not showed significant difference among the experimental groups,

421 different sensitivity to several molecules has been reported for the wide variety of cells from the IS,

422 leaving the possibility of other molecular interactions that escaped our control and awareness.

\section{Acknowledgments}

425 We thank M.V.Z Claudia Rivera-Cerecedo and her animal facility staff for assisting in the breeding

426 of experimental animals. We also thank Diana Millán-Aldaco and Marcela Palomero-Rivero for

427 their technical support. This work was supported by Grant IN-209719 and Grant IA 202919 , both

428 from Programa de Apoyo a Proyectos de Innovación Tecnológica (PAPIIT), Dirección General de

429 Asuntos del Personal Académico (DGAPA), Universidad Nacional Autónoma de México (UNAM);

430 to JMM and KENC, respectively. Grant FC-2016-2125 from Fronteras en la Ciencia, Consejo

431 Nacional de Ciencia y Tecnología (CONACYT), also to JMM. Margarita I Palacios-Arreola is a

432 Posdoctoral fellowship from DGAPA, UNAM. We also receive funding from Fideicomiso: bases de

433 colaboración “Transplantes al cerebro” to René Drucker Colín. In addition, Salvador Valencia- 
434 Sánchez received a complementary scholarship from Fondo de Estudios e Investigaciones Ricardo J.

435 Zevada, to complete the final stages of this study. Salvador Valencia-Sánchez is a doctoral student

436 from Programa de Doctorado en Ciencias Biomédicas, Universidad Nacional Autónoma de México

437 (UNAM) and received fellowship 223625 from CONACYT. 


\section{Author Contributions}

440 SVS performed the training of animals, the extraction of tissues, most of the experimental procedures

441 and the analysis of data, writing and edition of this manuscript.

442 KNC helped with the design of experiments regarding the proliferation assays and cytotoxicity.

443 MIP performed some of the experiments and analyzed some of the data regarding the asses of cell

444 subpopulations distributions.

$445 \mathrm{RD}$ collaborated in the direction of this work and the design of the experimental procedures. Also 446 contributed with reagents and the majority of expenses derived from this work.

447 OP collaborated in the direction of this work, the analysis of data and preparation of the draft. Also

448 contributed with reagents.

449 JMM participated in the direction of SVS, the design, analysis and writing of the present paper. Also 450 contributed with reagents, and all the flow cytometric analyses.

453 The authors declare that they have no conflict of interest. 


\section{References}

460 [1] R.V.T. Santos, É'. C. Caperuto, L.F.B.P. Costa Rosa, Effects of acute exhaustive physical 461 exercise upon glutamine metabolism of lymphocytes from trained rats, Life Sci. 80 (2007) 573-578. doi:10.1016/j.lfs.2006.10.015.

[2] D.J. Freidenreich, J.S. Volek, Immune responses to resistance exercise, Exerc. Immunol. Rev. 18 (2012) 8-41.

[3] D.J. Freidenreich, J.S. Volek, The Immune Response to Exercise, Elsevier Inc., 2013. doi:10.1016/B978-0-12-396454-0.00009-6.

[4] M. Gleeson, N.C. Bishop, D.J. Stensel, M.R. Lindley, S.S. Mastana, M.A. Nimmo, The antiinflammatory effects of exercise: mechanisms and implications for the prevention and treatment of disease, Nat. Rev. Immunol. 11 (2011) 607-615. doi:10.1038/nri3041. Moraes, P.O. Prada, D. Guadagnini, R.M. Marin, A.G. Oliveira, T.M. Augusto, H.F. Carvalho, exercise to hypothalamic insulin and leptin sensitivity through IKK $\beta$ and ER stress inhibition, PLoS Biol. 8 (2010) 31-32. doi:10.1371/journal.pbio.1000465. 

doi:10.14814/phy2.13070. , and Adaptation, Society. 80 (2000) 1055-1081. durations of exercise on macrophage functions in mice Effects of different durations of exercise on macrophage functions in mice, J. Appl. Physiol. (2001) 789-794.

[9] G. Lancaster, S. Halson, Q. Khan, P. Drysadale, F. Wallace, A.E. Jeukendrup, M.T. Drayson, M. Gleeson, Effects of acute exhaustive exercise and chronic exercise training on type 1 and type 2 T lymphocytes, Exerc Immunol Rev. 10 (2004) 91-104. http://www.researchgate.net/publication/8096824_Effects_of_acute_exhaustive_exercise_and _chronic_exercise_training_on_type_1_and_type_2_T_lymphocytes/file/79e4150c59a6b1619f .pdf. Cells and Monocytes: New Perspectives in HIV Pathogenesis, EBioMedicine. 6 (2016) 31-41. doi:10.1016/j.ebiom.2016.02.012. regulates T cell activation, differentiation, and functions, Front. Immunol. 6 (2015) 1-6. doi:10.3389/fimmu.2015.00001. 
[13] D. a. Padgett, R. Glaser, How stress influences the immune response, Trends Immunol. 24 (2003) 444-448. doi:10.1016/S1471-4906(03)00173-X.

501 [14] P.B. Sparling, a Giuffrida, D. Piomelli, L. Rosskopf, a Dietrich, Exercise activates the endocannabinoid system., Neuroreport. 14 (2003) 2209-11. doi:10.1097/01.wnr.0000097048.56589.47. Meeusen, Intense exercise increases circulating endocannabinoid and BDNF levels in humans _ Possible implications for reward and depression, Psychoneuroendocrinology. 37 (2012)

[16] G. Galdino, T.R.L. Romero, J.F.P. Silva, D.C. Aguiar, A.M. De Paula, J.S. Cruz, C. Parrella, F. Piscitelli, I.D. Duarte, V. Di Marzo, A.C. Perez, The endocannabinoid system mediates aerobic exercise-induced antinociception in rats, Neuropharmacology. 77 (2014) 313-324. human immune tissues and leukocyte subpopulations, Eur. J. Biochem. 61 (1995) 54-61.

[19] R. Tanasescu, C.S. Constantinescu, Cannabinoids and the immune system: an overview.,

[18] T.W. Klein, C. Newton, H. Friedman, Cannabinoid receptors and immunity., Immunol. Today. 19 (1998) 373-81. http://www.ncbi.nlm.nih.gov/pubmed/21873455.

519 [20] M.T. Cencioni, V. Chiurchiù, G. Catanzaro, G. Borsellino, G. Bernardi, L. Battistini, M. Maccarrone, Anandamide suppresses proliferation and cytokine release from primary human 
T-lymphocytes mainly via CB2 receptors, PLoS One. 5 (2010) 2-11. doi:10.1371/journal.pone.0008688.

523

[21] N. Malek, K. Popiolek-Barczyk, J. Mika, B. Przewlocka, K. Starowicz, Anandamide, acting via $\mathrm{CB} 2$ receptors, alleviates LPS-induced neuroinflammation in rat primary microglial cultures, Neural Plast. 2015 (2015). doi:10.1155/2015/130639.

[22] S. Radom-Aizik, F. Zaldivar, F. Haddad, D.M. Cooper, Impact of brief exercise on peripheral blood NK cell gene and microRNA expression in young adults, J. Appl. Physiol. 114 (2013) 628-636. doi:10.1152/japplphysiol.01341.2012.

[23] E. a Murphy, J.M. Davis, a S. Brown, M.D. Carmichael, N. Van Rooijen, a Ghaffar, E.P. Mayer, Role of lung macrophages on susceptibility to respiratory infection following shortterm moderate exercise training., Am. J. Physiol. Regul. Integr. Comp. Physiol. 287 (2004) R1354-8. doi:10.1152/ajpregu.00274.2004.

[24] G.-D. Syu, H.-I. Chen, C.J. Jen, Differential effects of acute and chronic exercise on human neutrophil functions., Med. Sci. Sports Exerc. 44 (2012) 1021-7. doi:10.1249/MSS.0b013e3182408639.

[25] H. Sugiura, H. Sugiura, H. Nishida, R. Inaba, S.M. Mirbod, H. Iwata, Effects of different durations of exercise on macrophage functions in mice Effects of different durations of exercise on macrophage functions in mice, J. Appl. Physiol. (2012) 789-794.

[26] Y. Saito, Y. Kusaka, M. Shimada, Effects of exercise intensity on circulating leukocyte subpopulations., Environ. Health Prev. Med. 8 (2003) 18-22. doi:10.1007/BF02897939.

[27] J.M. Davis, E. a Murphy, a S. Brown, M.D. Carmichael, a Ghaffar, E.P. Mayer, Effects of moderate exercise and oat beta-glucan on innate immune function and susceptibility to 
respiratory infection., Am. J. Physiol. Regul. Integr. Comp. Physiol. 286 (2004) R366-R372. doi:10.1152/ajpregu.00304.2003.

545

546

[28] A.L. Slusher, T.M. Zúñiga, E.O. Acevedo, Maximal Exercise Alters the Inflammatory Phenotype and Response of Mononuclear Cells, 2017. doi:10.1249/MSS.0000000000001480.

[29] C.E. Matthews, I.R. a S. Ockene, P.S. Freedson, M.C. Rosal, P. a Merriam, J.R. Hebert, Moderate to vigorous physical activity and risk of upper-repiratory tract infection, Med. Sci. Sport. Exerc. 34 (2002) 1242-1248. doi:10.1097/00005768-200208000-00003.

[30] L.M. Parsons, D. Denton, G. Egan, M. Mckinley, R. Shade, J. Lancaster, P.T. Fox, Neuroimaging evidence implicating cerebellum in support of sensorycognitive processes associated with thirst, 97 (2000).

[31] L.T. MacKinnon, Special feature for the Olympics: effects of exercise on the immune system: overtraining effects on immunity and performance in athletes., Immunol. Cell Biol. 78 (2000) 502-9. doi:10.1111/j.1440-1711.2000.t01-7-.x.

[32] A. Córdova, A. Sureda, J.A. Tur, A. Pons, Immune response to exercise in elite sportsmen during the competitive season, J. Physiol. Biochem. 66 (2010) 1-6. doi:10.1007/s13105-0100001-2.

[33] Y.S. Lin, M.S. Jan, H.I. Chen, The effect of chronic and acute exercise on immunity in rats. / Effet de 1' exercice chronique et aigu sur 1' immunite chez des rats., Int. J. Sports Med. 14 (1993) 86-92. http://search.ebscohost.com/login.aspx $?$ direct $=$ true $\& d b=$ sph \&AN=SPH360924\&site=ehostlive.

[34] a K. Blannin, L.J. Chatwin, R. Cave, M. Gleeson, Effects of submaximal cycling and long- 
term endurance training on neutrophil phagocytic activity in middle aged men., Br. J. Sports Med. 30 (1996) 125-129. doi:10.1136/bjsm.30.2.125.

[35] V. Chiurchiu, L. Battistini, M. Maccarrone, Endocannabinoid signalling in innate and adaptive immunity, Immunology. 144 (2015) 352-364. doi:10.1111/imm.12441.

[36] W.E. Greineisen, H. Turner, Immunoactive effects of cannabinoids: considerations for the therapeutic use of cannabinoid receptor agonists and antagonists., Int. Immunopharmacol. 10 (2010) 547-55. doi:10.1016/j.intimp.2010.02.012.

S. Galgglje, S. Mary, J. Marchand, D. Dussossoy, D. Carrikre, P. Camyon, M. Bouaboula, D. Shirez, G.L.E. Fur, P. Casellas, Expression of central and peripheral cannabinoid receptors in human immune tissues and leukocyte subpopulations, Signals. 61 (1995) 54-61.

[38] M. Bouaboula, M. Rinaldi, P. Carayon, C. Carillon, B. Delpech, D. Shire, G. Le Fur, P. cannabinoid CB 2 receptor mRNA in mouse immune cell subpopulations and following B cell 
587 Supplementary figure legends

589 Supplementary Fig 1. Gating strategy for the flow cytometric analysis of rat spleen 590 subpopulations and their expression of CB1 and CB2 receptors.

591 Single cell suspension was prepared and stained with fluorochrome-conjugated antibodies to separate splenocyte subpopulations and to mark cannabinoid receptors (CB1 and CB2). Data was analyzed with FlowJo software 8.7 for Mac. Lymphocytes were identified by their scatter properties (FSC-A x SSC-A plot). Splenocyte subpopulations were characterized by surface staining and gated for their quantity assessment. Subsequently each cellular subpopulation was analyzed for their expression of both cannabinoid receptors in their surface.

Supplementary fig 2. Levels of IgG are not affected by chronic exercise.

600 The analysis of total IgG was assessed for every experimental group with the use of a direct semi-

601

602 quantitative ELISA. Statistical analysis did not show any significant difference among the experimental groups: SED (shaded bar), TC (white bar) and EXE (Solid bar). P $>0.05$. ANOVA, $603 \mathrm{p}=0.0676, \mathrm{n}=6$.

Supplementary fig 3. Serum corticosterone concentration one day after the last exercising bout.

606 Data is shown as mean $(\mathrm{ng} / \mathrm{ml})+$ - SE for each group. There was no significant difference among group values in concentration of serum corticosterone. Groups analyzed: SED (shaded bar), TC

608 (white bar) and EXE (Solid bar). When data was statistically analyzed (ANOVA, n=6, p=0.0473, 609 Tukey's) results show a significant difference among the experimental groups. Nonetheless, The post 610 hoc analysis did not show any significant interaction among the experimental groups. Means of the 611 groups: TC and EXE (102.6 and $96.5 \mathrm{ng} / \mathrm{ml}$ respectively) were notoriously higher than that of the 612 SED group $(46.8 \mathrm{ng} / \mathrm{ml})$. 
A
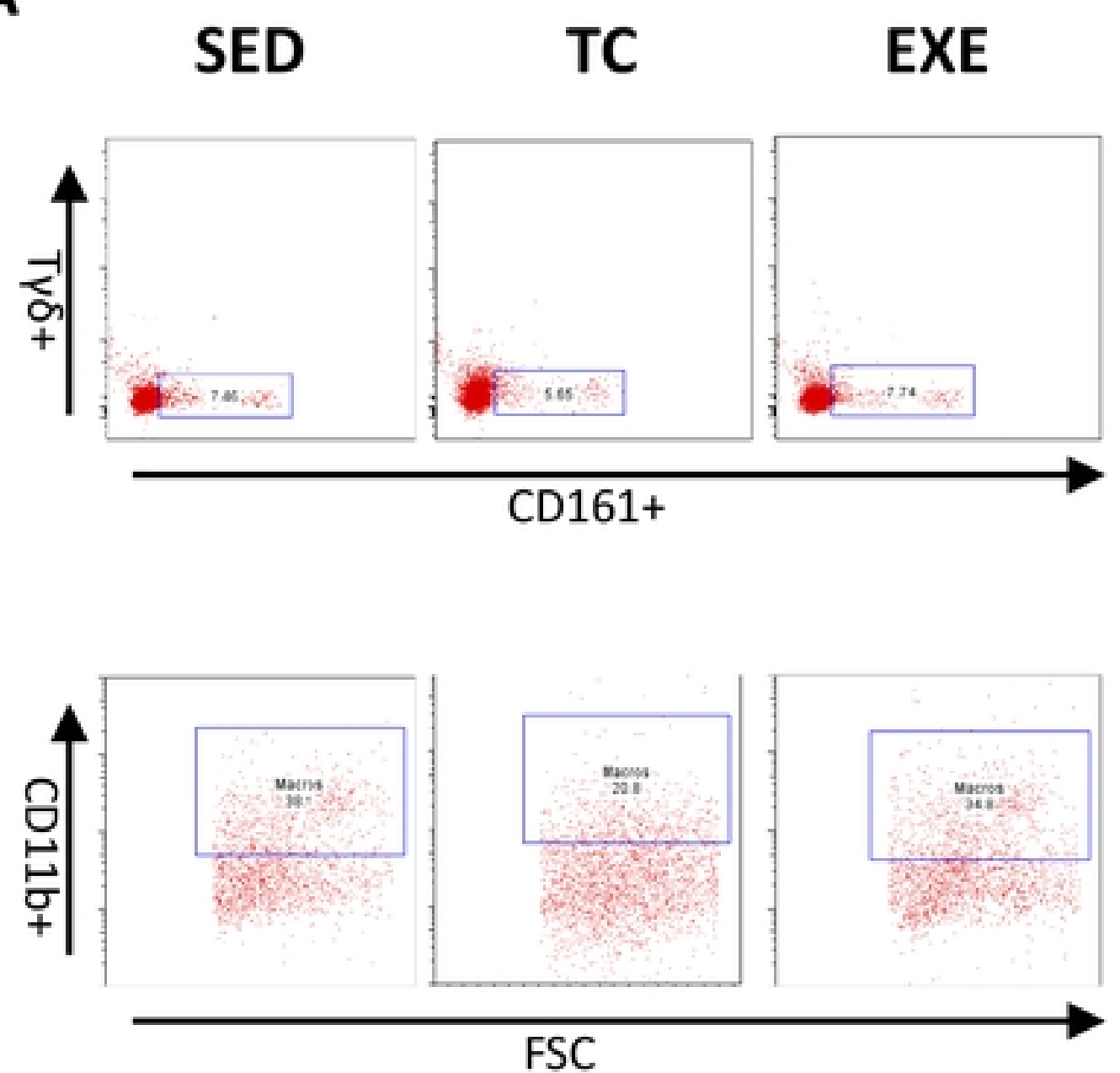

B

\% CD161+

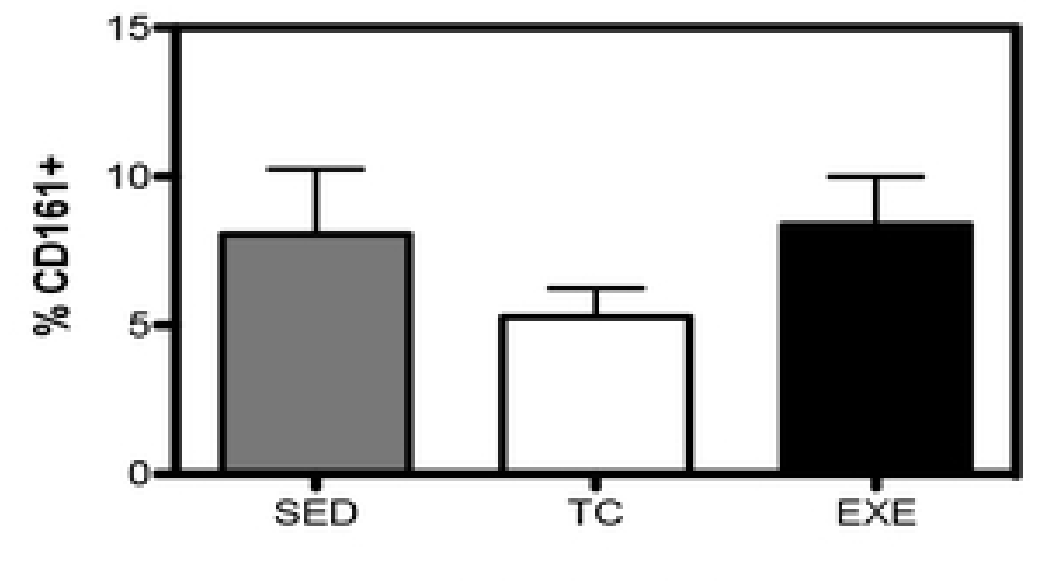

$\%$ CD11b+

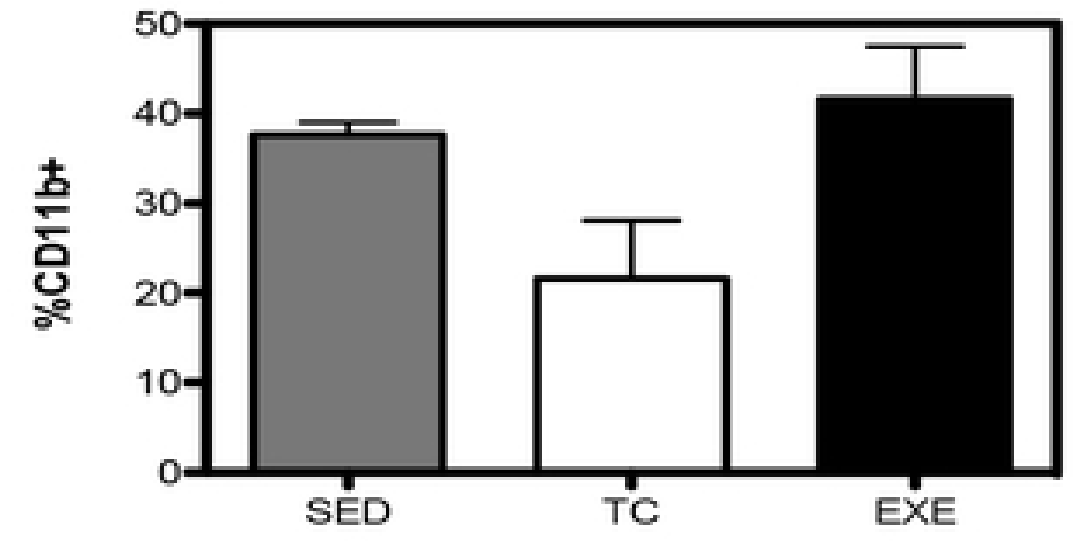

Figure 1 


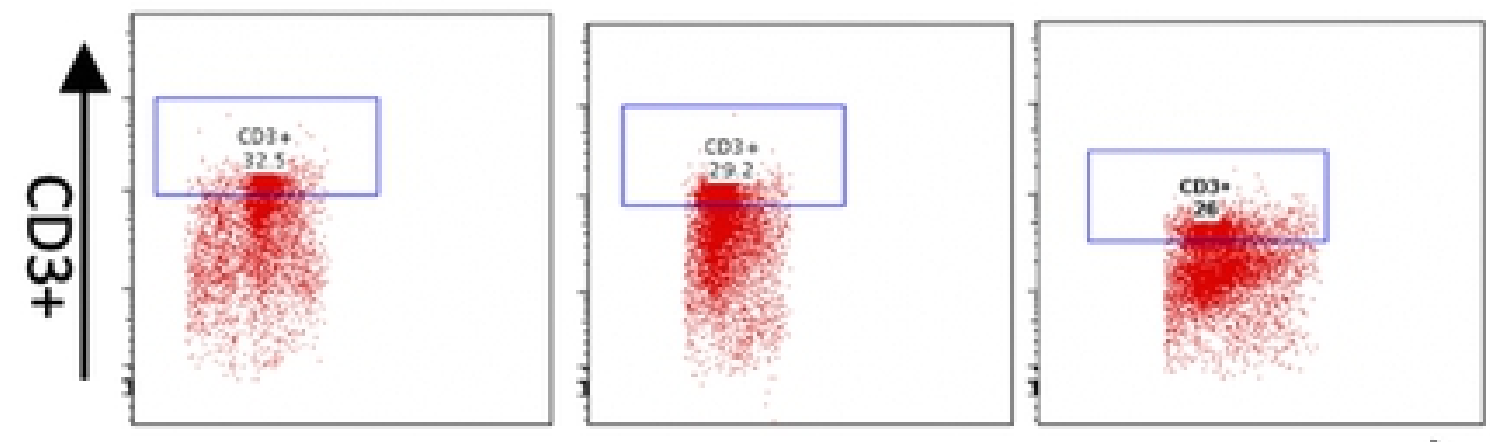

bioRxiv preprint doi- https://doiora/10 1101/708792: this version posted July 19 2019. The copyright holder forthis preprint (which was not
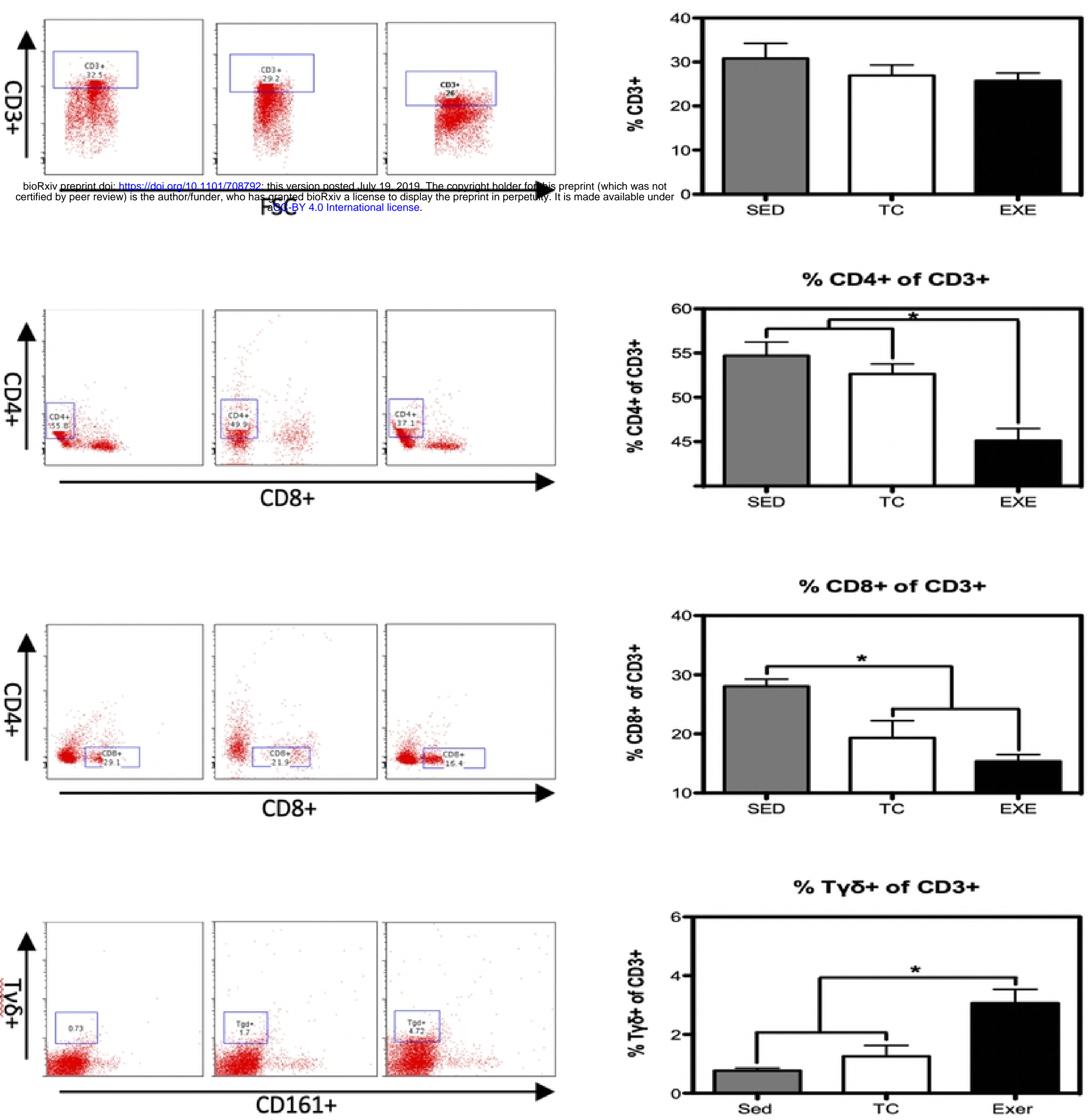

Figure 2 
SED

TC
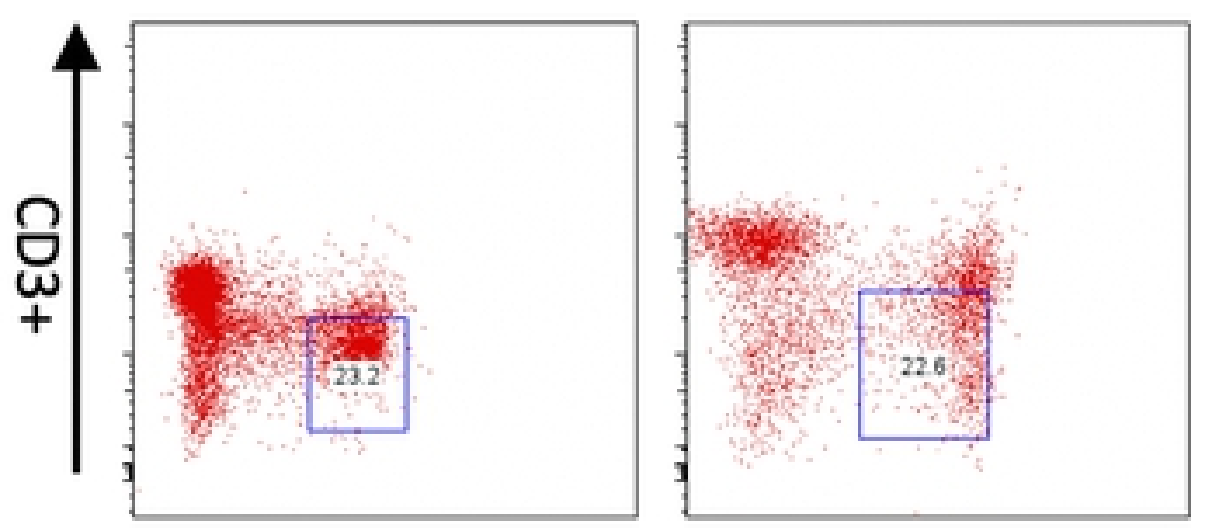

CD45 RA+
EXE

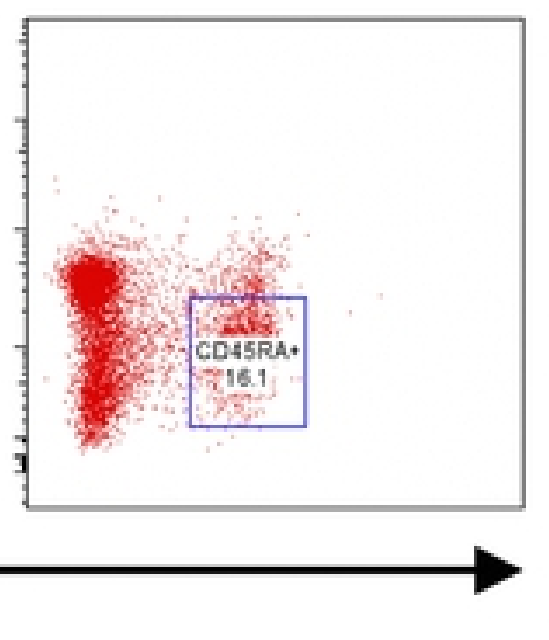

$\%$ CD45 RA+

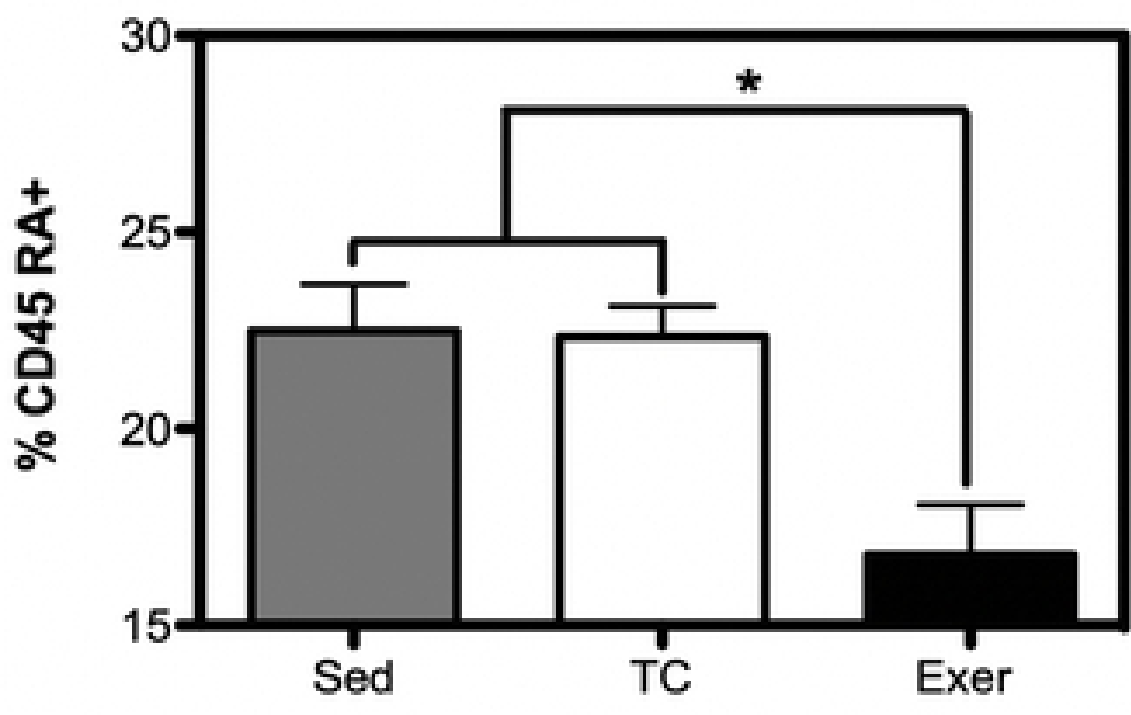

Figure 3 
CBR in Natural killers

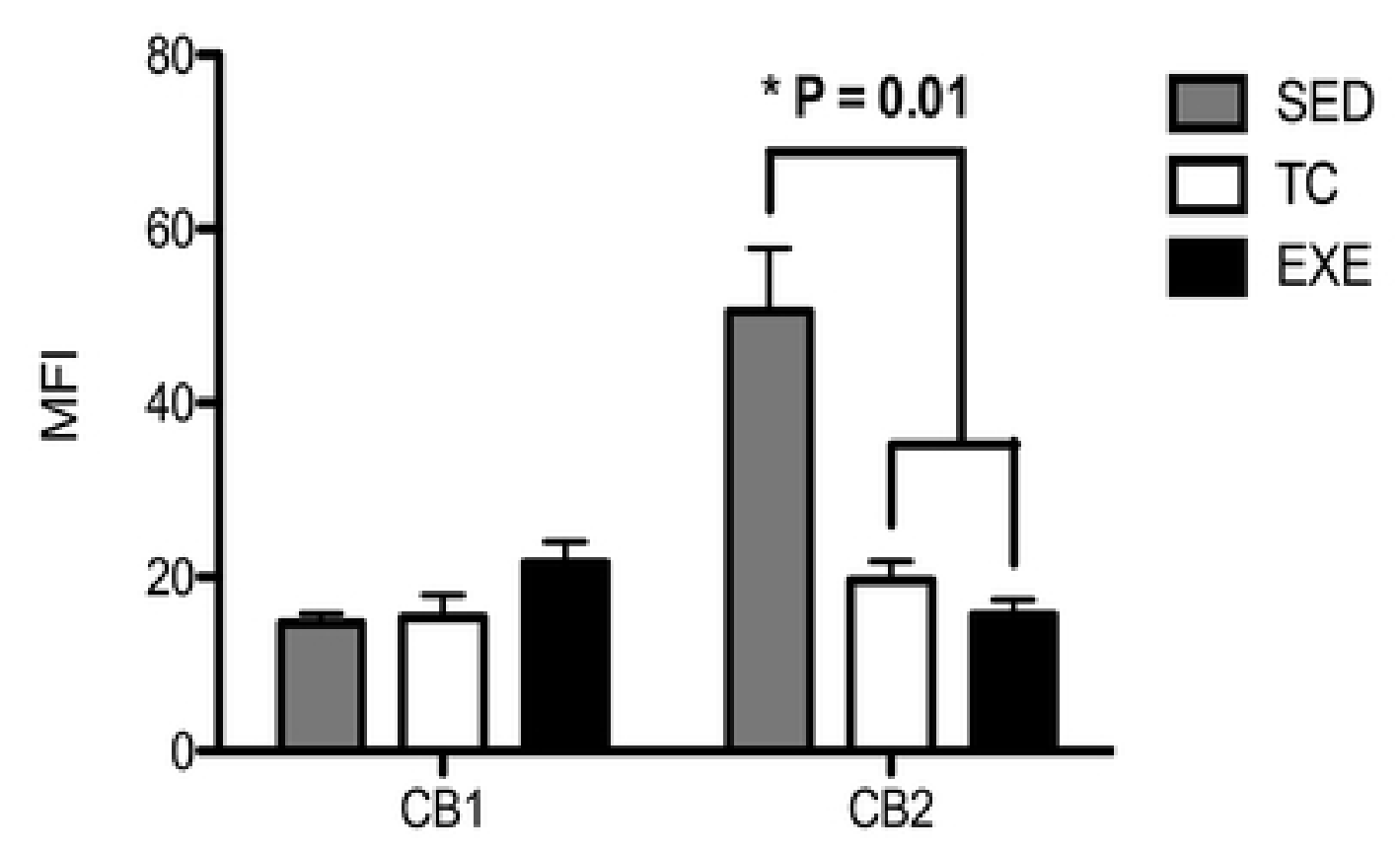

CBR in Macrophages

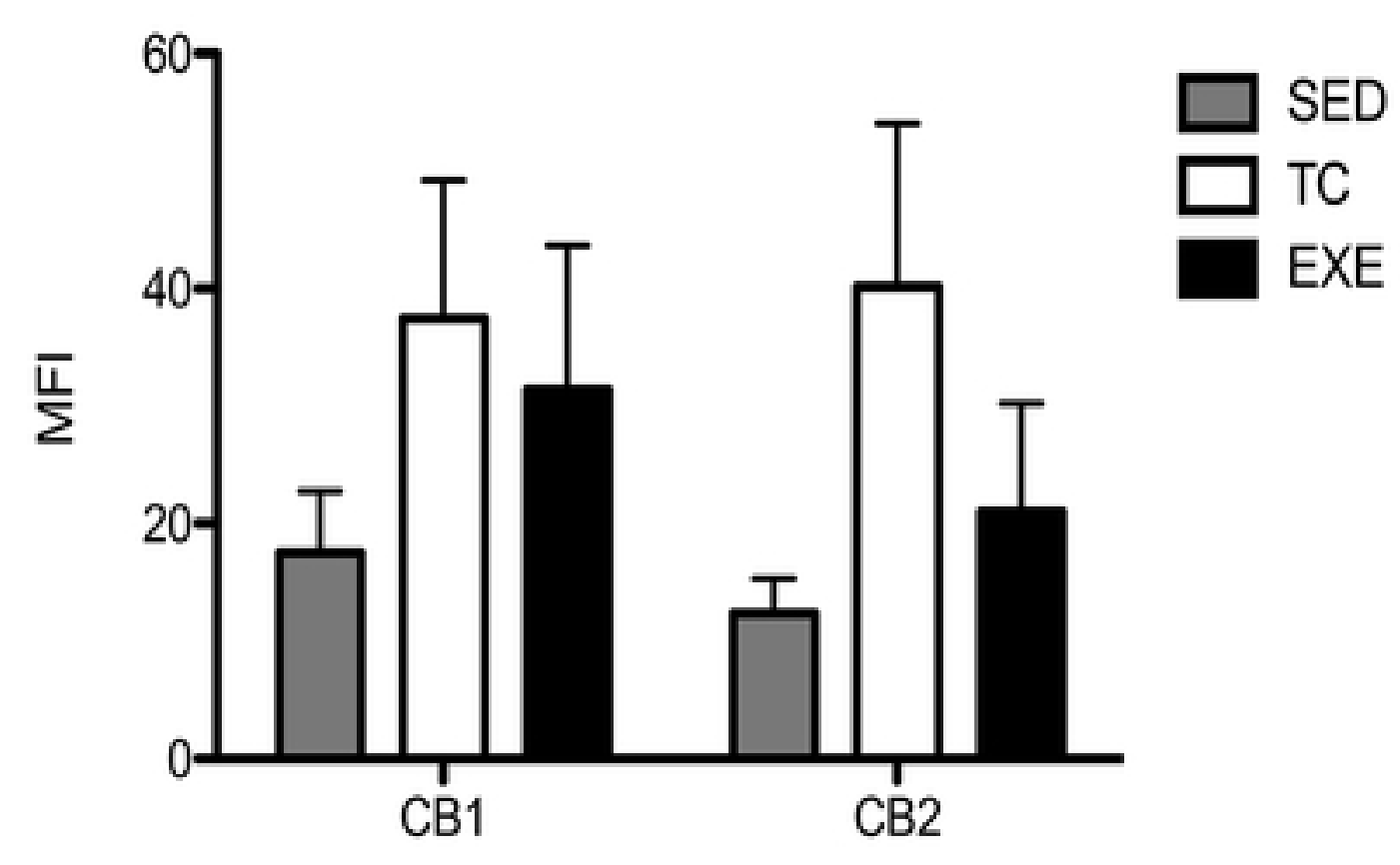

Figure 4 

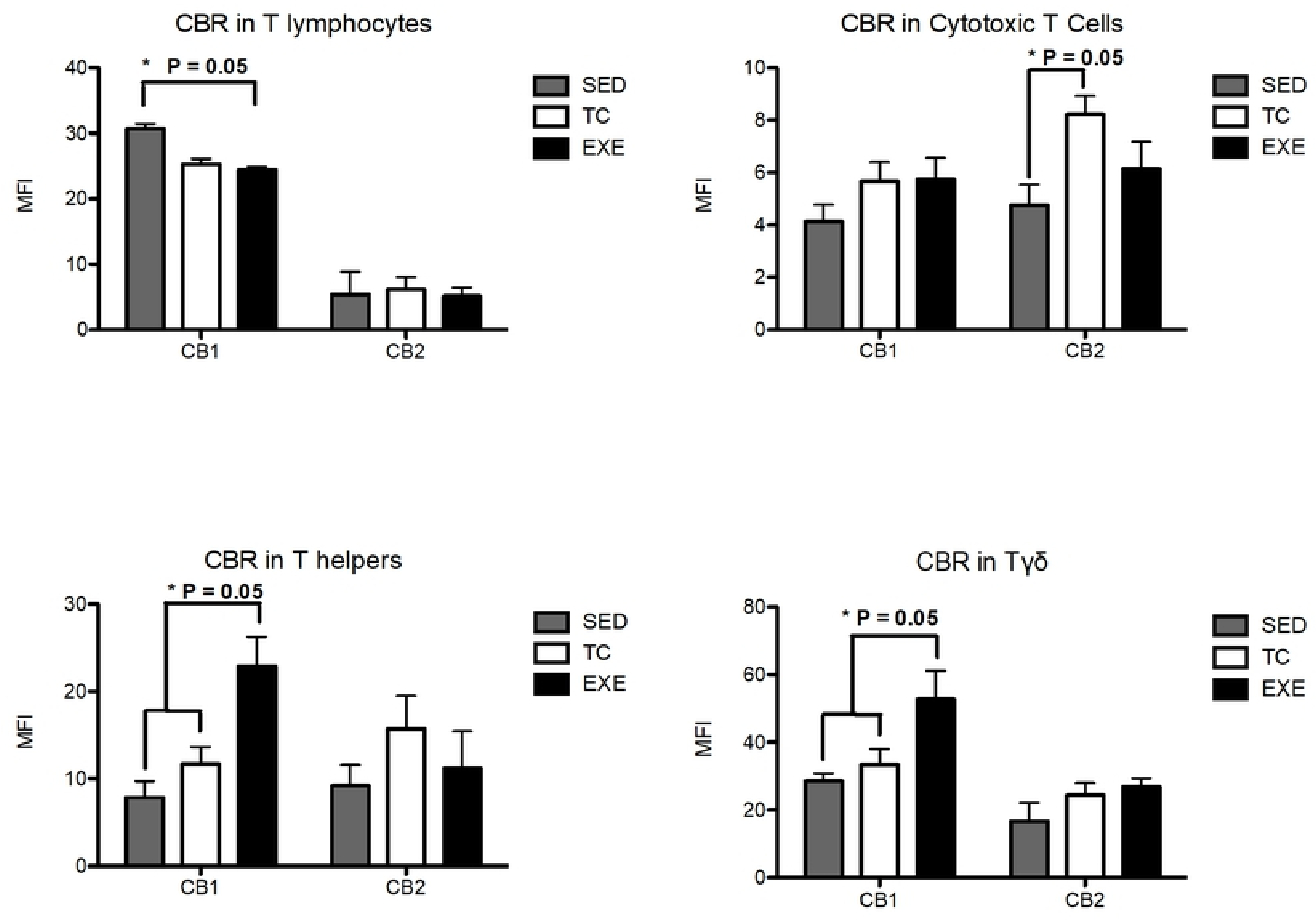

Figure 5 


\section{CBR in B Lymphocytes}

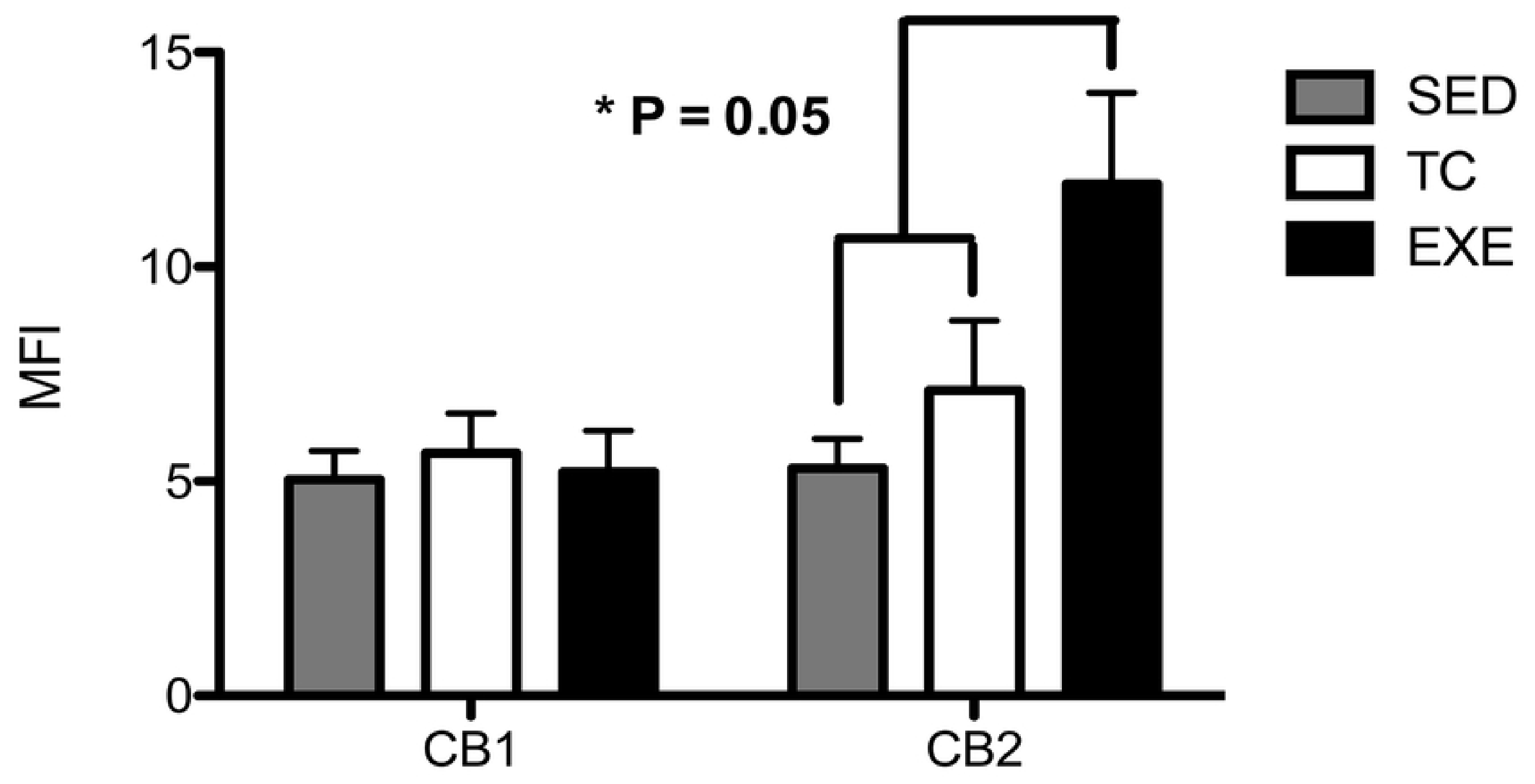

Figure 6 


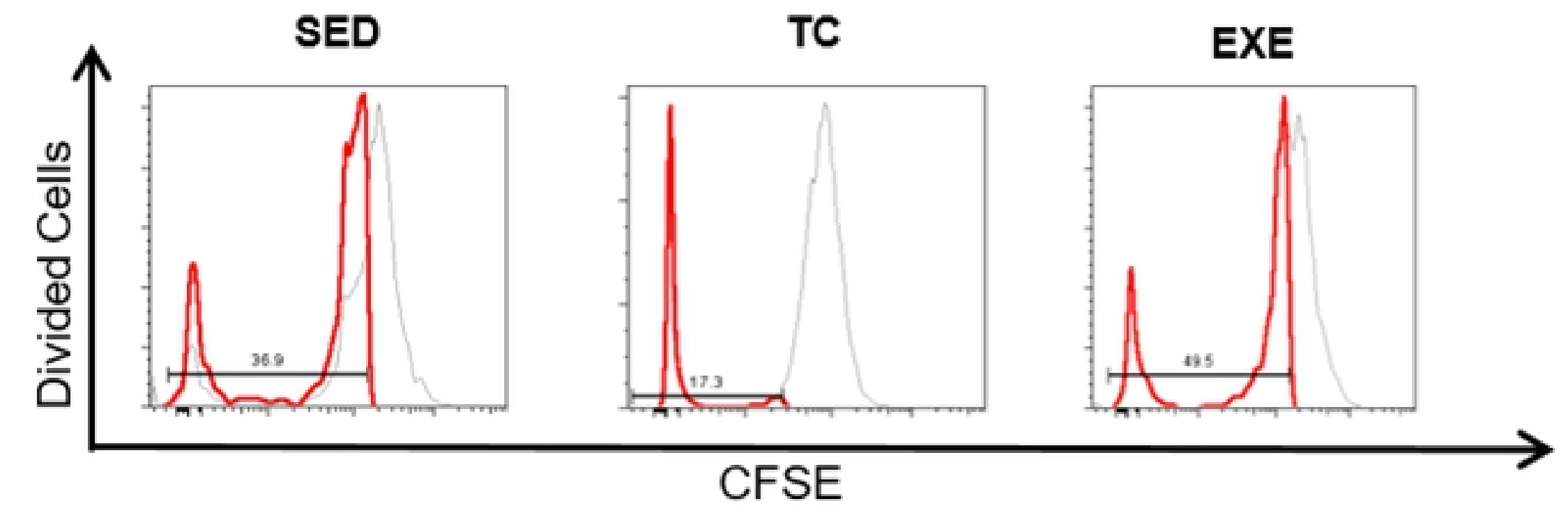

B

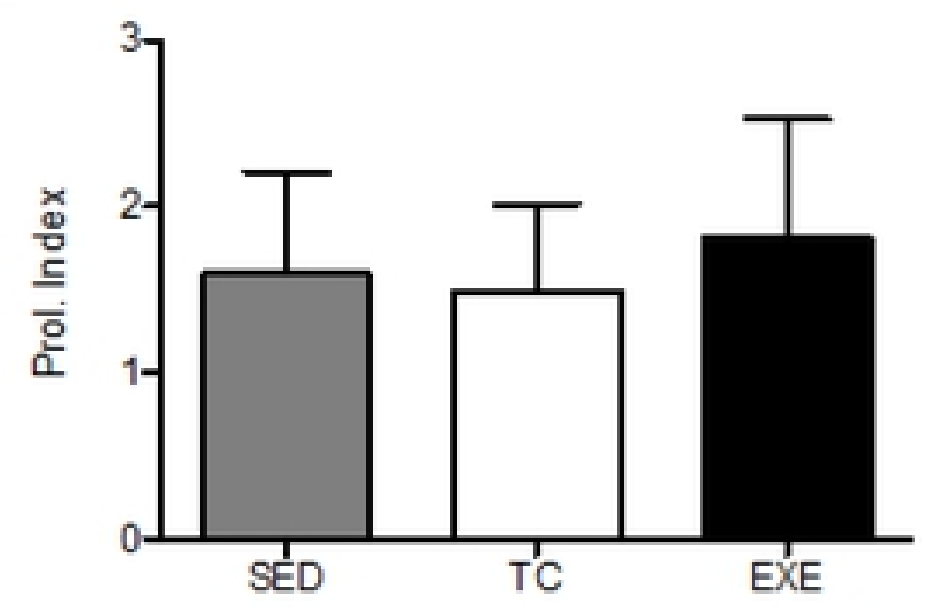

D
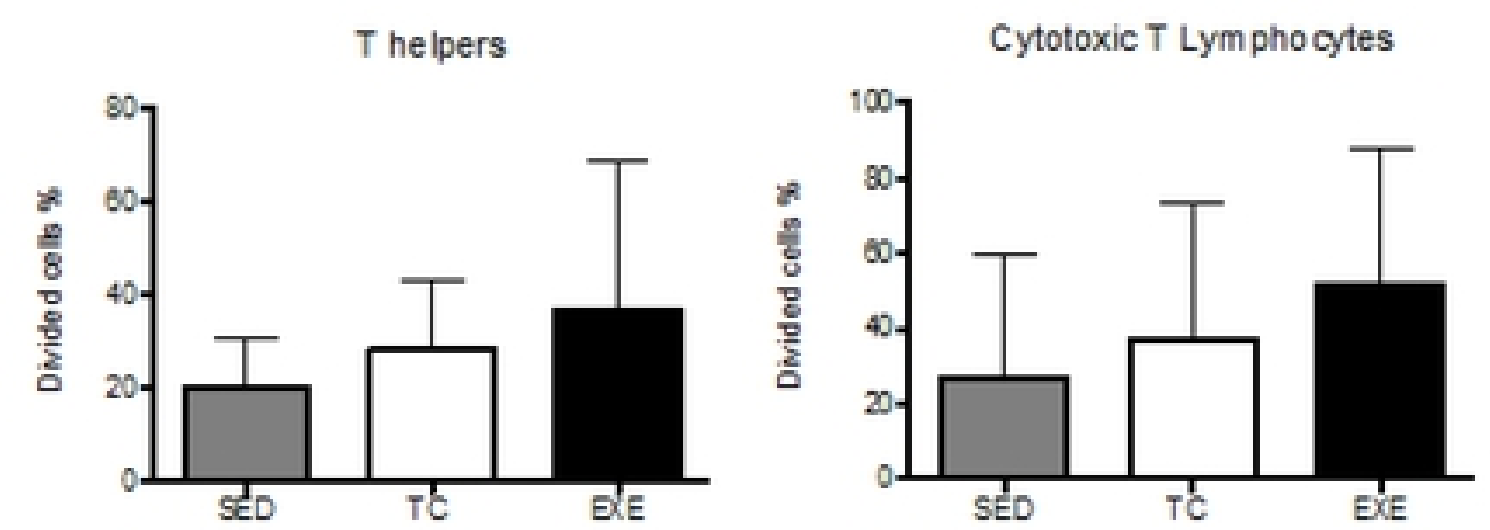

C
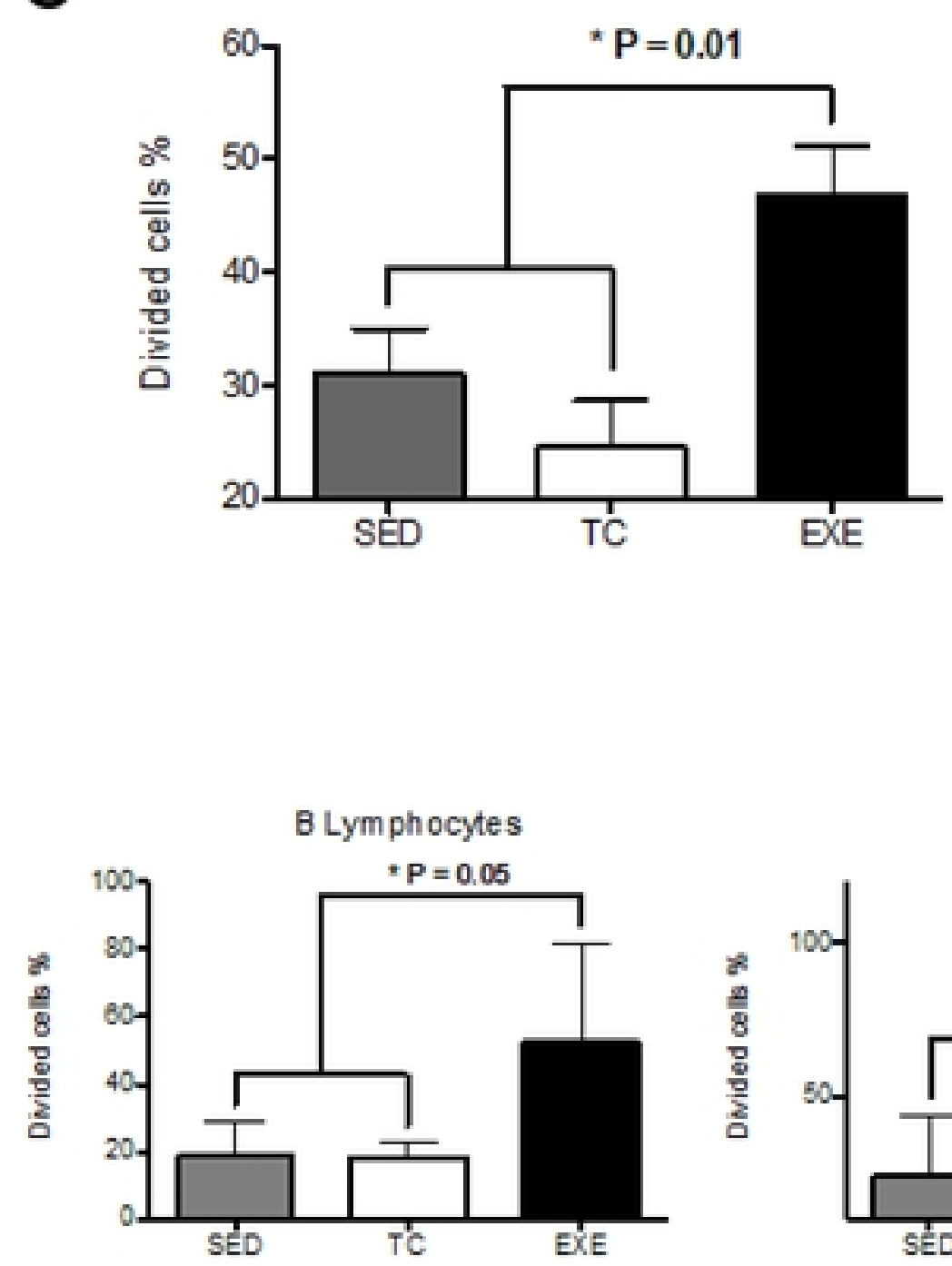

Natural Killers

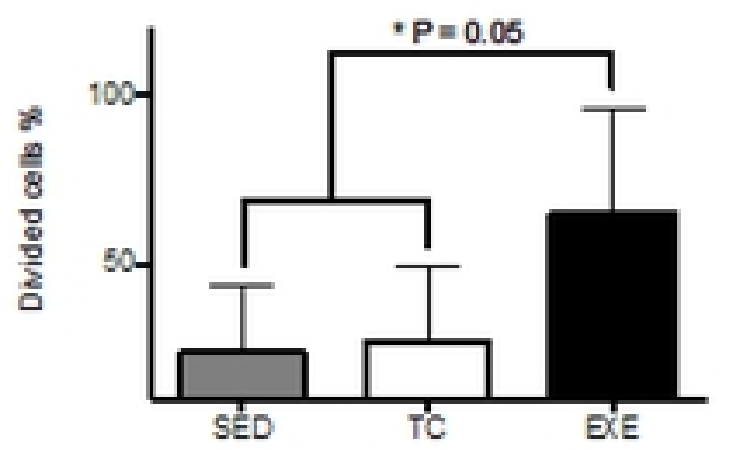

Figure 7 

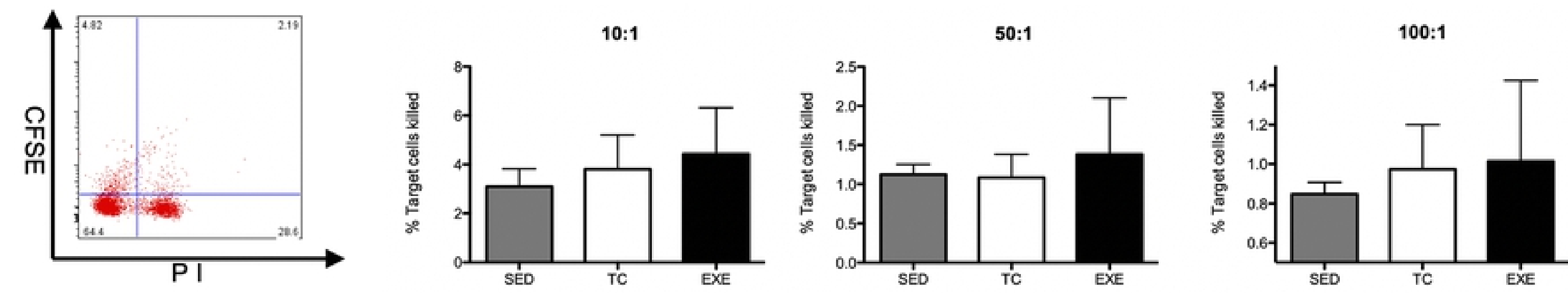

Figure 8 